University of Wollongong

Research Online

Faculty of Engineering and Information

Faculty of Engineering and Information

Sciences - Papers: Part B

Sciences

2017

\title{
Single step fabrication of antimicrobial fibre mats from a bioengineered protein-based polymer
}

André Da Costa

University of Minho

Ana Pereira

University of Minho

A C. Gomes

University of Minho

José C. Rodríguez-Cabello

Universidad de Valladolid

Vitor Sencadas

University of Wollongong, victors@uow.edu.au

See next page for additional authors

Follow this and additional works at: https://ro.uow.edu.au/eispapers1

Part of the Engineering Commons, and the Science and Technology Studies Commons

Research Online is the open access institutional repository for the University of Wollongong. For further information contact the UOW Library: research-pubs@uow.edu.au 


\title{
Single step fabrication of antimicrobial fibre mats from a bioengineered protein- based polymer
}

\author{
Abstract \\ Genetically engineered protein polymers functionalized with bioactive domains have potential as \\ multifunctional versatile materials for biomedical use. The present work describes the fabrication and \\ characterisation of antimicrobial fibre mats comprising the antimicrobial elastin-like recombinamer (ELR) \\ CM4-A200. The CM4-A200 protein polymer derives from the genetic fusion of the ABP-CM4 antimicrobial \\ peptide from Bombyx mori with 200 repetitions of the pentamer VPAVG. This is the first report on non- \\ crosslinked fibre mats fabricated with an antimicrobial ELR stable in solution. Thermal gravimetric \\ analysis of CM4-A200 fibre mats shows one single degradation step at temperatures above $300{ }^{\circ} \mathrm{C}$, with \\ fibres displaying a higher thermal degradation activation. The electrospun CM4-A200 fibres display high \\ antimicrobial activity against Gram-positive and Gram-negative bacteria with no detectable cytotoxic \\ effects against normal human skin fibroblasts and keratinocytes, revealing the great potential of these \\ polymers for the fabrication of biomedical materials.

\section{Disciplines} \\ Engineering | Science and Technology Studies

\section{Publication Details} \\ da Costa, A., Pereira, A. M., Gomes, A. C., Rodriguez-Cabello, J. C., Sencadas, V., Casal, M. \& Machado, R. \\ (2017). Single step fabrication of antimicrobial fibre mats from a bioengineered protein-based polymer. \\ Biomedical Materials, 12 (4), 045011-1-045011-11.
}

\section{Authors}

André Da Costa, Ana Pereira, A C. Gomes, José C. Rodríguez-Cabello, Vitor Sencadas, Margarida Casal, and Raul Machado 


\title{
Single step fabrication of antimicrobial fibre mats from a bioengineered protein-based polymer
}

\author{
A da Costa $^{1}$, A M Pereira ${ }^{1}$, A C Gomes ${ }^{1}$, J C Rodriguez-Cabello ${ }^{2,3}$, V Sencadas ${ }^{4,5,6}$, M \\ Casal $^{1} * \#$ and R Machado ${ }^{1 * \#}$ \\ ${ }^{1}$ CBMA (Centre of Molecular and Environmental Biology), Department of Biology, University \\ of Minho, Campus de Gualtar, 4710-057 Braga, Portugal \\ ${ }^{2}$ Bioforge (Group for Advanced Materials and Nanobiotechnology), Edificio LUCIA, \\ Universidad de Valladolid, Valladolid, Spain \\ ${ }^{3}$ Networking Research Centre on Bioengineering, Biomaterials and Nanomedicine (CIBER- \\ $B B N)$, E-47011 Valladolid, Spain \\ ${ }^{4}$ Centro/Departamento de Física, University of Minho, Campus de Gualtar, 4710-057 Braga, \\ Portugal \\ ${ }^{5}$ School of Mechanical, Materials and Mechatronics Engineering, University of Wollongong, \\ Wollongong, NSW 2522, Australia \\ ${ }^{6}$ ARC Centre of Excellence for Electromaterials Science, University of Wollongong, NSW
} 2522, Australia

\# - Both authors contributed equally and are listed in alphabetical order

* Corresponding authors:

Raul Machado

raulmachado@bio.uminho.pt

Margarida Casal

mcasal@bio.uminho.pt 


\begin{abstract}
Genetically engineered protein polymers functionalized with bioactive domains offer potential as multifunctional versatile materials for biomedical use. The present work describes the fabrication and characterization of antimicrobial fibre mats comprising the antimicrobial elastin-like recombinamer CM4-A200. The CM4-A200 protein polymer derives from the genetic fusion of the ABP-CM4 antimicrobial peptide from Bombyx mori with 200 repetitions of the pentamer VPAVG. This is the first report on noncrosslinked fibre mats fabricated with an antimicrobial elastin-like recombinamer stable in solution. Thermal gravimetric analysis of CM4-A200 fibre mats shows one single degradation step at temperatures above $300{ }^{\circ} \mathrm{C}$, with fibres displaying a higher thermal degradation activation. The electrospun CM4-A200 fibres display high antimicrobial activity against Gram-positive and Gram-negative bacteria with no detectable cytotoxic effects against normal human skin fibroblasts and keratinocytes, revealing the great potential of these polymers for the fabrication of biomedical materials.
\end{abstract}

Keywords: Recombinamers, elastin-like polypeptide, antimicrobial activity, electrospinning, antimicrobial peptide.

Running head: Antimicrobial fibre mats of a bioengineered protein-based polymer 


\section{Introduction}

Within the materials used for biomaterials manufacturing, natural polymers attract great attention due to the superior biocompatibility and low immunogenicity when compared with synthetic polymeric materials. Recombinant protein-based polymers (rPBPs) are biopolymers comprising tandem repetitions of amino acid sequences, by using the knowledge obtained from the observation of natural proteins [1]. These protein polymers are produced by recombinant DNA technology, allowing to precisely control their molecular structure. This potential can be explored to create customized materials by incorporating functionalities that otherwise would not be present [2-5]. Elastin-like recombinamers (ELRs) are elastin-mimetic rPBPs with amino acid sequence deriving from the hydrophobic domain of tropoelastin. Typically, ELRs consist of repeats of the pentamer (VPGXG), where X, the guest residue, is any amino acid except proline [6]. The most remarkable features of ELRs include the ability to undergo reversible, temperature-dependent, hydrophobic assembly from aqueous solution [6] and extraordinary good biocompatibility [7-9]. However, the high solubility of ELR materials in aqueous solutions at temperatures below their transition temperature, hampers their usage in the development of new biomaterials. In general, the structural integrity of ELR materials can be maintained by including lysine residues in the guest residue to provide free amine groups to react with the crosslinking agent [10-12]. For instance, electrospun fibre mats of an ELR with sequence VPGKG were structurally stabilized via a two-step method involving a vapor-phase and further aqueous completion of crosslinks by using glutaraldehyde [11]. Similarly, electrospun fibre mats of ELRs containing cell adhesion domains were stabilized via crosslinking through the lysine residues of the VPGKG sequence, using genipin [13] or HDMI/acetone solution [14]. Still, the uncontrolled chemical crosslinking via lysine residues can lead to a loss of properties in ELR-based polypeptides with bioactive domains enriched in such residues. In addition, the extensive crosslinking within the VPGXG may preclude its proper folding leading to a loss of properties. Other fibre stabilization procedures involve blending the ELR with other polymers such as polycaprolactone [15] and methacrylate [16]. Nevertheless, these approaches may exert a negative impact in the bioactivity and mechanical properties of the ELR fibres. An alternative to the crosslinking step is to explore the thermal hysteresis of poly(VPAVG). The pentamer VPAVG is unique among ELRs, self-assembling at temperatures above $33{ }^{\circ} \mathrm{C}$ but only disassembles when the temperature is strongly cooled down to $\sim 12{ }^{\circ} \mathrm{C}[3,17-19]$. This 
preserves the structural integrity of ELR-based materials within a broad range of temperatures.

As the development of antimicrobial materials is a rapidly growing field, particularly for the biomedical field, there is a demand for new versatile materials [20]. This is particularly relevant due to the advent of multidrug resistance in bacterial pathogens. The overuse and misuse of antibiotics over decades have exerted an unprecedented selective pressure on bacteria resulting in multi-antibiotic resistant organisms [21, 22]. This represents a major danger to public health as it threatens our ability to treat common infectious diseases with conventional antimicrobials, resulting in prolonged illness and an economic burden in healthcare settings [23, 24].Consequently, great efforts are put into the research of new and effective antimicrobials as well as in infection prevention strategies that do not require antibiotics [25]. Antimicrobial peptides (AMPs) are considered a promising alternative to tackle multidrug resistant bacteria [26-28]. This class of small immunomodulatory peptides is found in almost all living organisms, representing the first line of defence of multicellular organisms as part of their innate immune system [26, 29]. Typically, AMPs are characterized by small molecular weight, cationic net charge, amphipathic structure and broad antimicrobial activity against bacteria, fungi and viruses [28]. Another remarkable feature of AMPs is that they act against bacteria but are essentially harmless to mammalian cells [29, 30]. This selectivity towards bacterial cells and the nature of the antimicrobial mechanism highlight the potential of AMPs for innovative antimicrobial therapies.

Our team developed the genetically engineered polymer CM4-A200 [4] which combines in the same macromolecule the antimicrobial peptide ABP-CM4, isolated from the Chinese silkworm [31], and the ELR (VPAVG) 200 [17]. In this work we report the fabrication of stable antimicrobial CM4-A200 fibre mats without the need of crosslinking agents, demonstrating its potential use for skin tissue engineering and wound healing applications. 


\section{Methods}

\section{Materials:}

The recombinant CM4-A200 protein was obtained by heterologous production using Escherichia coli as cell factory [4]. Pure lyophilized CM4-A200 was completely dissolved in formic acid (FA, $\geq 98 \%$, MERCK) for a final concentration of $20 \%$ (w/v). The solutions were maintained at ice-cold temperature, dissolved with the help of a magnetic stirrer until complete dissolution, and allowed to set at room temperature (RT) for 30 min before processing.

\section{Sample preparation:}

Fibres. A custom-build electrospinning apparatus was used for the fabrication of fibrous membranes. The $20 \%(\mathrm{w} / \mathrm{v})$ polymer solution was transferred to a plastic syringe fitted with a steel needle with an inner diameter of $0.5 \mathrm{~mm}$. Electrospinning was conducted with an electric field ranging from $1.35 \mathrm{kV} . \mathrm{cm}^{-1}$, applied with a high voltage power supply from Gamma High Voltage. A syringe pump (KDScientific) was used to feed the polymer solutions into the needle tip at a feed rate of $0.1 \mathrm{~mL} \cdot \mathrm{h}^{-1}$ and the electrospun fibre mats were collected in a static grounded aluminium plate, placed at 15 $\mathrm{cm}$ from the needle tip. The as-spun fibres were stored for at least $72 \mathrm{~h}$ before any characterization to allow complete solvent evaporation.

Films. In some cases, free standing films were produced by solvent casting using $10 \%$ (w/v) CM4-A200 formic acid solution and cast on polytetrafluoroethylene substrates with $10 \mathrm{~mm}$ diameter [4]. The solvent was evaporated at RT for $48 \mathrm{~h}$ under extraction.

\section{Morphological analysis:}

The morphology of the fibre membranes was observed under a scanning electron microscope (SEM, FEI Nova 200) with an accelerating voltage of $3 \mathrm{kV}$. The average fibre diameter was calculated with at least 100 randomly selected fibres from different micrographs, using ImageJ image processing software [32].

Fourier transform infrared spectroscopy with attenuated total reflectance (ATR-FTIR): FTIR spectra from $4000 \mathrm{~cm}^{-1}$ to $600 \mathrm{~cm}^{-1}$ were acquired at room temperature with a Bruker Tensor 27 in ATR mode. The spectra were collected with a resolution of $2 \mathrm{~cm}^{-1}$ after 64 scans. 
Thermal analysis:

Thermal changes were assessed by differential scanning calorimetry measurements (DSC) and thermogravimetric analysis (TGA). DSC experiments were carried with a Mettler Toledo DSC 823e with liquid nitrogen as cooler. The samples were cut into small pieces from the middle region, and placed into $50 \mu \mathrm{l}$ aluminium pans followed by a heating step from $35{ }^{\circ} \mathrm{C}$ to $200{ }^{\circ} \mathrm{C}$ with a constant heating rate of $10{ }^{\circ} \mathrm{C} \cdot \mathrm{min}^{-1}$. The thermal degradation kinetics were assessed in a Perkin-Elmer Pyris 1 TGA apparatus with different heating rate scans (from $10{ }^{\circ} \mathrm{C}$ to $40{ }^{\circ} \mathrm{C} \cdot \mathrm{min}^{-1}$ ), under a nitrogen atmosphere. Analysis of the collected data was performed using the OriginPro software (OriginLab, Northampton, MA). All samples were air-dried for at least $48 \mathrm{~h}$ prior to any characterization study.

Degree of swelling and hydrolytic degradation:

The degree of swelling for the CM4-A200 fibres was assessed by measuring the difference in weight between dry and swollen samples. Fibre mats were immersed in PBS 1x ( NaCl 8 g, KCl 0.2 g, $\mathrm{Na}_{2} \mathrm{HPO}_{4} 1.44$ g, $\mathrm{KH}_{2} \mathrm{PO}_{4} 0.24$ g, per litre, at $\left.\mathrm{pH} 7.4\right)$ at RT and collected at regular intervals. At each time point, the samples were taken after immersion and the excess of liquid was gently removed with filter paper. The degree of swelling was then calculated using Equation 1:

$$
\text { Degree of swelling }(\%)=\left(\frac{W s-W d}{W d}\right) \times 100
$$

where $W s$ is the mass of the swollen sample and $W d$ is the initial dry mass. Each value was averaged from three independent measurements.

The hydrolytic degradation of the fibres was evaluated in PBS 1x solution. The samples were immersed in PBS 1x (15 mL) and incubated at $37^{\circ} \mathrm{C}$ for different time periods, with PBS renewal every $72 \mathrm{~h}$. After the incubation time, the samples were washed with ultrapure water to remove PBS and air-dried at RT until reaching a constant mass. The extent of hydrolytic degradation was calculated by weighing the samples before and after incubation in an analytical microbalance (Mettler Toledo, error $\pm 0.1 \mathrm{mg}$ ) according to Equation 2. All measurements were performed in triplicate. 


$$
\% M L=\left(1-\frac{M f}{M i}\right) \times 100
$$

where $M L$ is mass loss in percentage, $M f$ is the sample mass after the incubation time and $M i$ is the initial sample mass.

\section{Evaluation of antibacterial activity:}

The antimicrobial activity of the electrospun fibres was assessed using a modified protocol based on a previously described assay [4]. Prior to cell incubation, the fibres were cut into $100 \mathrm{~mm}^{2}$ samples and sterilized by UV exposure for $30 \mathrm{~min}$ at a wavelength of $254 \mathrm{~nm}$. The fibres were then transferred to sterile 24 well plates and a stainless steel nut was placed on top of the samples to delimitate the area of inoculation and to avoid material bending. Fibre samples were inoculated with $15 \mu \mathrm{l}$ of bacterial cell suspension at a concentration of $1 \times 10^{7} \mathrm{CFUs} / \mathrm{mL}$, followed by incubation at $37^{\circ} \mathrm{C}$ for 30 and $120 \mathrm{~min}$. After incubation, $1 \mathrm{~mL}$ of sterile PBS was added and carefully agitated prior plating on LB agar for bacterial CFUs enumeration. Pseudomonas aeruginosa ATCC10145 and Staphylococcus aureus ATCC6538 were used as a model Gram-negative and a Gram-positive bacteria, respectively and $100 \mathrm{~mm}^{2}$ aluminium foil squares were used as negative control. All measurements were performed in triplicate and results expressed as \% kill using Equation 3:

$$
\% \text { kill }=\frac{(\text { Control CFUs-Sample CFUs })}{\text { Control CFUs }} \times 100
$$

For SEM analysis, the fibres were incubated for 120 min with $15 \mu \mathrm{l}$ of a $1 \times 10^{7}$ $\mathrm{CFUs} / \mathrm{mL}$ bacterial cell suspension at $37^{\circ} \mathrm{C}$. Sterile polystyrene (PS) disks were used as control. After incubation, the culture media was removed, $1 \mathrm{~mL}$ of $2.5 \% \mathrm{v} / \mathrm{v}$ glutaraldehyde in PBS was added to each sample and maintained at $37^{\circ} \mathrm{C}$ for $1 \mathrm{~h}$. Immediately after, the fibre samples were rinsed with $1 \mathrm{~mL}$ of distilled water and dehydrated using sequential ethanol-water dilutions with increasing percentages of ethanol (55.0 \%, $70.0 \%, 80.0 \%, 90.0 \%, 95.0 \%$ and $100.0 \%$ v/v of ethanol). Samples were submerged in $0.5 \mathrm{~mL}$ of each solution for $30 \mathrm{~min}$ at RT. Finally, the samples were dried at $37^{\circ} \mathrm{C}$ for a period of $30 \mathrm{~min}$ prior to coating with $\mathrm{Au} / \mathrm{Pd}$ using a sputter coater and analysed by SEM (NanoSEM - FEI Nova 200). 


\section{Cell culture and cytotoxicity evaluation:}

NCTC 2544 (human keratinocytes) and BJ-5ta (telomerase-immortalized normal human skin fibroblasts) cell lines were obtained from the American Type Culture Collection (ATCC, through LGC standards, Teddington, Middlesex, UK). Cells were cultured at $37^{\circ} \mathrm{C}, 5 \% \mathrm{CO}_{2}$, in humidified environment and according to ATCC recommendations: BJ-5ta medium - 4 parts of Dulbecco's modified Eagle’s medium containing 4 mM Lglutamine, $4.5 \mathrm{~g} / \mathrm{L}$ glucose, $1.5 \mathrm{~g} / \mathrm{L}$ sodium bicarbonate, and 1 part of Medium 199, supplemented with $10 \%$ (v/v) of foetal bovine serum (FBS), $1 \%$ (v/v) penicillin/streptomycin solution and $10 \mu \mathrm{g} / \mathrm{mL}$ hygromycin B; NCTC medium - $93 \%$ (v/v) Dulbecco’s modified Eagle’s medium containing 4 mM L-glutamine, 4.5 g/L glucose, $1.5 \mathrm{~g} / \mathrm{L}$ sodium bicarbonate, supplemented with $7 \%$ (v/v) of FBS.

The materials' cytotoxicity was assessed in BJ-5ta and NCTC 2544 cell lines. UV sterilized fibre samples of approximately $100 \mathrm{~mm}^{2}$ were incubated with $750 \mu \mathrm{L}$ of cell culture medium without FBS for $24 \mathrm{~h}$ at $37^{\circ} \mathrm{C}, 5 \% \mathrm{CO}^{2}$ in a humidified environment. In parallel, $100 \mu \mathrm{L}$ of a cell suspension of $6.6 \times 10^{4}$ cells/mL were seeded and cultured in surface treated 96-well plates (Nunclon polystyrene 96-well MicroWell, Thermo Scientific) for $24 \mathrm{~h}$ under the conditions described above. Following incubation, the cell culture medium was removed and replaced with the medium conditioned by the $24 \mathrm{~h}$ contact with the fibre samples. Cell viability was then evaluated after 48 and $72 \mathrm{~h}$, using the MTS assay (CellTiter $96^{\circledR}$ Aqueous One Solution Cell Proliferation, Promega) according to the manufacturer's instructions. Cells cultured in standard culture medium and in $30 \%$ DMSO (Sigma-Aldrich) were used as positive and negative controls for cell viability, respectively. Results were expressed as percentage of viability in relation to the positive control (set as $100 \%$ viability).

\section{Statistics and data analysis:}

One-way analysis of variance (ANOVA) with Bonferroni's post-test was carried out using GraphPad Prism 5 Software to compare the means of the different data sets within each experiment. A value of $P<0.05$ was considered to be statistically significant. All experiments were performed in triplicate. 


\section{Results}

\section{Morphology and structural characterization:}

Figure 1 shows the surface morphology of CM4-A200 electrospun fibres. The fibres were randomly and isotropically distributed and characterized by smooth, cylindrical and non-defective fibres with an average diameter of $149 \pm 71 \mathrm{~nm}$. In order to evaluate if the processing technique induces structural modifications, the electrospun fibres were analysed by ATR-FTIR and compared with the pure lyophilized sample (figure 2). The infrared spectra of the fibre mats reveals the characteristic absorption bands of amide groups centred at $1628 \mathrm{~cm}^{-1}$ for amide I (mainly C=O stretching vibration), $1523 \mathrm{~cm}^{-1}$ for amide II (out-of-phase combination of $\mathrm{N}-\mathrm{H}$ bending and C-N stretching vibrations) and $1231 \mathrm{~cm}^{-1}$ for amide III (in-phase combination of N-H bending and C-N stretching vibrations) [33, 34]. The broad absorption band observed in the $3500-3100 \mathrm{~cm}^{-1}$ band region is attributed to contributions from amide A ( $\mathrm{N}-\mathrm{H}$ stretching vibration) and free water present in the material due to the storage conditions [33]. The same pattern was found for the lyophilized sample, suggesting that electrospinning did not induce significant structural modifications. Furthermore, the absence of the characteristic infrared peaks of formic acid at 1750 and $2942 \mathrm{~cm}^{-1}$ (Data from the National Institute of Standards and Technology) reveals the total evaporation of the solvent during sample processing and storage.

\section{Figure 1 -}

\section{Figure 2:}

\section{Thermal stability and degradation}

Thermal characterization, including determination of the activation temperature, was assessed in the fibre mats and further compared with free standing films of CM4-A200 produced by solvent casting. The DSC thermograms of the electrospun fibres and free standing films are characterized by a single broad endothermic peak centred at $\sim 85^{\circ} \mathrm{C}$, starting at the initial temperature and extending to $\sim 150{ }^{\circ} \mathrm{C}$ (figure 3a). For both fibres and films, the TGA thermogram is characterized by two major weight loss steps (figure 3b): i) the first, at temperatures below $150{ }^{\circ} \mathrm{C}$, is associated to the loss of moisture [35] 
due to the handling and storage of the sample at room conditions and proved to be independent of the processing technique, corresponding to a weight loss of $\sim 8 \%$ and; ii) the second degradation process starts at $\sim 300{ }^{\circ} \mathrm{C}$ and goes up to $450{ }^{\circ} \mathrm{C}$ and is related to protein degradation, namely progressive deamination, decarboxylation and depolymerisation arising from the break of polypeptide bonds [35, 36]. Regarding the initial step of weight loss, a broader band is observed in the TGA from films, when compared to the fibres. This result can be explained by the thicker matrix of the films that leads to a slower loss of water. Figure 3c depicts the derivative thermogravimetric curve (DTG) profiles of CM4-A200 fibres and films. The peaks observed in both graphs corroborate the two steps of degradation described above and give indications of the mean temperature for each thermal step.

Protein thermal stability was characterized by the kinetic parameters of the thermal degradation. The Kissinger mathematical model [37, 38] was used to determine the activation energy from plots of the logarithm of the heating rate $v s$ the inverse of temperature maximum reaction rate, in constant heating rate $(\beta)$ experiments:

$\ln \left(\frac{\beta}{T_{p}^{2}}\right)=\frac{\ln \left(A E_{a c t}\right)}{R}+\ln \left[n\left(1-\alpha_{p}\right)^{1-n}\right]-\frac{E_{a c t}}{R T_{p}}$

where $A$ is the Arrhenius pre-exponential factor $\left(\mathrm{min}^{-1}\right), R$ is the ideal gas constant (8.31 $\mathrm{J} . \mathrm{mol}^{-1} \cdot \mathrm{K}^{-1}$ ), and $E_{\text {act }}$ is the activation energy of the thermal degradation process, where $T_{p}$ and $\alpha_{p}$ are the peak temperature and the conversion at the maximum weight loss rate [39].

Figure 3d, represents the plots of $\ln \left(6 / T_{p}{ }^{2}\right)$ versus $1 / T_{p}$ for fibres and films with a linear regression line fitted to the data. The slope of the line equals $-E_{a c t} / R$, and allows the determination of the activation energy for the different materials. Accordingly, the activation energy obtained for the fibre mats was higher than that calculated for the films, with values of 184 and $166 \mathrm{~kJ} \cdot \mathrm{mol}^{-1}$, respectively.

\section{Figure 3:}

Hydrolytic degradation and swelling degree: 
The stability and the water uptake capability (degree of swelling) of the electrospun membranes was evaluated by immersing the fibre mats in PBS for different time intervals at $37^{\circ} \mathrm{C}$ while measuring changes in weight (figure 4). After the time course of 30 days, the electrospun fibre mats revealed low degradation with a corresponding mass loss of $13 \pm 10 \%$ (figure $4 \mathrm{a}$ ). The swelling of the fibres was very fast, reaching values of $611 \pm 117 \%$ after only 5 min of immersion in PBS (figure 4b). After the initial rapid swelling, the fibres maintained its behaviour for the remaining time of the assay, with a final degree of swelling of $679 \pm 50 \%$.

\section{Figure 4.}

\section{Antimicrobial activity:}

The electrospun mats were tested for their antimicrobial activity against $P$. aeruginosa, a Gram-negative bacteria, and S. aureus, a Gram-positive bacteria by a direct contact assay. As observed in figure 5a, the electrospun mats display antimicrobial activity against both $P$. aeruginosa and $S$. aureus after 120 min of incubation, causing $95.9 \pm 1.6$ $\%$ and $77.0 \pm 2.6 \%$ of kill, respectively. Regarding P. aeruginosa, 30 min of incubation with the fibres was sufficient to achieve a \% of kill of $99.0 \pm 0.1 \%$, highlighting the strong antimicrobial effect of the material. The antimicrobial activity of the fibres was time-dependent for $S$. aureus, as the killing efficiency rose from $0.4 \pm 1.7 \%$, at $30 \mathrm{~min}$ of incubation, to $77 \pm 2.6 \%$, after 120 min of incubation.

SEM micrographs of $P$. aeruginosa and $S$. aureus cells after 120 min of contact with the fibre mats clearly show altered morphology, presenting a collapsed form and an apparent reduction of size (figure 5b). The loss of the fibre-like shape in the electrospun mats is likely a consequence of the cell fixation protocol that involves a sequence of several dehydration/hydration steps that could result in fibre merging.

\section{Figure 5 -}

\section{Cytotoxicity:}

Cell viability in response to the fibre mats was assessed by indirect contact with BJ-5ta (fibroblasts) and NCTC 2544 (keratinocytes) cell lines (figure 6). Cytotoxicity 
evaluation on keratinocytes showed values of $88.4 \pm 2.2 \%$ of cell viability after $24 \mathrm{~h}$ of incubation, while no significant cytotoxicity was found for fibroblasts, with values of $99.0 \pm 1.4 \%$ of cell viability. However, no significant cytotoxicity was detected after 72 h of contact, with the keratinocytes reaching $90.9 \pm 4.9 \%$ of cell viability and the fibroblasts even suggesting a slight proliferative effect with $105.3 \pm 3.1 \%$ (figure 6).

\section{Figure 6 -}

\section{Discussion}

In this study, we describe the fabrication of antimicrobial, defective-free CM4-A200 electrospun fibres from a $20 \%(\mathrm{w} / \mathrm{v})$ protein polymer solution prepared in formic acid. Previously, we have demonstrated that CM4-A200 films produced by solvent casting display their best antimicrobial activity when obtained from formic acid solutions [4]. In addition, formic acid demonstrated to be a suitable solvent, yielding a clear solution without any evidence of gelation, not altering the secondary structure of the protein polymer [4]. Therefore, the choice of the solvent was based on its ability to dissolve the recombinant protein while preserving the antimicrobial activity after processing. The produced fibres were cylindrical in shape with an average fibre diameter of $149 \pm$ $71 \mathrm{~nm}$ (figure 1), which is the lowest reported to date for an ELR. Previously, Benitez et al. [11] demonstrated that electrospun ribbons from an ELR functionalized with a RGD domain could be produced from solutions with concentrations of $25 \mathrm{wt} \%$ and $35 \mathrm{wt} \%$ in deionized water. These ribbon-like fibres presented an average fibre diameter of $1.2 \pm$ $0.3 \mu \mathrm{m}$ and $1.8 \pm 0.4 \mu \mathrm{m}$, for 25 and 35 wt\%, respectively. Similarly, García-Arévalo et al. [14] described the production of electrospun ribbon-like fibres with diameters around $800 \mathrm{~nm}$ from a $25 \mathrm{wt} \%$ aqueous ELR solution. More recently, Putzu et al. [13] reported the fabrication of electrospun fibres from an ELR containing cell adhesion sequences prepared in 2,2,2-trifluoroethanol. The authors tested the effect of several experimental electrospinning parameters in fibre shape and width, demonstrating that fibres with an average diameter of $180 \pm 57.7 \mathrm{~nm}$ could be obtained from a $20 \%$ (w/v) protein solution. In the present work, the CM4-A200 fibres with a substantially smaller 
diameter, and their cylindrical morphology, resemble the ECM (figure 1a). These differences are most likely attributed to the conditions used for electrospinning (solvent and concentration), but also to the different amino acid composition found in CM4A200.

During electrospinning, the protein solution is subjected to electrostatic and stretching forces that may induce conformational changes. FTIR analysis allowed to determine if the processing technique influences the structure of the recombinant CM4-A200. As demonstrated in figure 2, both the lyophilized form and fibres present similar spectra with the characteristic amid bands of proteins. The absence of dislocations in peak position or changes in peak width or shape suggests that no significant structural changes occur during solution preparation and subsequent processing by electrospinning.

The differing dimensional architecture of films and fibres may give rise to different properties. For instance, while electrospinning generates nanofibrous porous mats with high porosity and high surface area-to-volume ratio [40], solvent casting typically leads to the production of thin films in a 2D dimensional arrangement with low porosity [41]. The thermal properties and thermal degradation kinetics are among the most important characterization techniques of polymeric materials to establish the temperature working range without loss of its properties. Differential scanning calorimetry was employed to study the thermal properties of dry CM4-A200 films and fibres (figure 3a), whereas the thermal degradation (figures $3 b$ and 3c) and the associated kinetics (figure 3d) were assessed by thermal gravimetric analysis. The broad endothermic peaks encountered in the DSC thermograms are related to the evaporation of bound water [35, 42]. The absence of any other peaks in the DSC thermograms suggests that the type of processing, either solvent casting or electrospinning, does not significantly affect the thermal properties of the material and that these samples remain stable over a wide range of temperatures. Analysis of the thermogravimetric curves (figure 3b) revealed two main stages of weight loss. The initial step of weight loss observed at temperatures below $150{ }^{\circ} \mathrm{C}$ is associated to the loss of moisture and correlates with the broad endothermic peak observed in the DSC experiment [35]. Due to the different structural organization of fibres and films, the peak corresponding to the evaporation of water is broader in the films, extending to higher temperatures. This relates with the more confined matrix of the material that hinders water evaporation. The main thermal decomposition stage starting at $\sim 300{ }^{\circ} \mathrm{C}$ corresponds to the effective stage of thermal 
degradation of the tested materials and is consistent with the degradation of other protein-based materials [35, 43, 44]. The main differences between the two materials are related with the different heating rates and are the base for the thermal degradation kinetic analysis (figure 3d). The higher activation energy obtained for the electrospun mats is most likely attributed to the higher polymer chain alignment along the longitudinal direction of the protein fibre, promoted by the electrospinning process. This same behaviour can be observed when comparing the activation energies obtained for PLLA films and electrospun membranes [39, 45].

Considering skin topical applications such as wound healing promotion, the determination of parameters such as water uptake capability and hydrolytic degradation are of utmost importance due to the moisturizing nature of the wound bed. As such, the hydrolytic degradation profile and swelling properties of the fibre mats were studied to understand their behaviour under humidified conditions (figure 4). Despite the great interest in novel ELR-based fibrous materials for biomedical devices, there is always the need to stabilize the structure of the fibre mats by means of a crosslinking agent. In fact, uncrosslinked ELR-based electrospun mats based on the VPGXG sequence are not structurally stable, even at temperatures above its coacervation temperature, quickly disaggregating in solution [14]. Remarkably, due to the intrinsic thermal hysteresis behaviour of (VPAVG) 200 , the CM4-A200 electrospun fibre mats were stable in solution without the need of crosslinking agents to promote structure stabilization. Indeed, the electrospun mats showed a slow degradation over the time course of the experiment with only $13 \%$ of weight loss after the 30 days of incubation. Nevertheless, this contrasts with the $1.8 \%$ of weight loss previously found for CM4-A200 films [4] and can be explained by the higher surface-to-volume ratio of the electrospun fibres when compared with films, that provides more surface availability to contact with the solution molecules [46], and enhance the hydrolytic degradation process. Regarding the swelling properties, the results obtained for the electrospun fibre mats are in the range of other electrospun rPBP [47].

The antimicrobial activity of CM4-A200 fibre mats was tested against bacteria with high clinical significance, namely P. aeruginosa (Gram-negative) and S. aureus (Grampositive). These bacteria belonging to the ESKAPE group are leading causes of nosocomial infections [22] and represent a major threat in skin-related infections and chronic wounds [48-50]. The electrospun fibres showed a highly efficient antimicrobial activity against both bacteria, reaching $\sim 100 \%$ kill against P. aeruginosa and $~ 77 \%$ kill 
for S. aureus, after 120 min of cells contact. The difference found for the antimicrobial activity in S. aureus is most likely due to the ability of the bacterial cells to form aggregates, reducing the direct contact with the fibres [4]. In opposition to antibiotics, which act in specific intracellular targets, AMPs mostly interact with the negatively charged bacterial cell membranes through electrostatic forces, inducing physical changes and damaging the biological membranes [26-28, 51]. Although not being clearly understood, the antimicrobial action of ABP-CM4 is generally accepted to be a consequence of an interaction with the cell membrane, leading to the formation of transmembrane pores that result in cell death [52]. This action at the cell is in agreement with our results suggesting that the antimicrobial activity of the fibres is a timedependent event that implies a direct contact with the bacterial cells. In fact, we have previously demonstrated that the antimicrobial activity of CM4-A200 based materials is not Gram-specific, is mediated by direct contact and not related with diffusion of the AMP [4].

Cytotoxicity to human cells is an important parameter to be evaluated during the validation of biomedical materials. In this work, cytotoxicity was evaluated in vitro by indirect contact (exposure to leachables) using the MTS assay, which gives an indication of cell metabolic activity. Despite the low hydrolytic degradation observed in PBS, the tested leachables were prepared with culture medium supplemented with fetal bovine serum, which contains several proteases that can degrade the protein-based materials [4]. Results of the exposure of BJ-5ta (fibroblasts) and NCTC 2544 (keratinocytes) cell lines to the leachables of the fibre mats revealed no significant cytotoxicity after $72 \mathrm{~h}$ of contact. In fact, a slight proliferative effect was even observed for the BJ-5ta cell line after $72 \mathrm{~h}$ of contact. This effect was previously observed in other studies and attributed to the presence of the $\operatorname{ELR}[4,19]$. In the future, the possible application of this material for wound healing will be considered as chemically synthesized elastin-like recombinamers (ELRs), based on the sequence VPAVG, showed very good biocompatibility in rats after intravitreal injection in the right eye and subcutaneous injection in the hind paw [53]. Also, an ELR based on the sequence VPAVG showed no haemolytic activity in fresh blood collected from mice [54]. Finally, a chemically synthesized ABP-CM4 did not display haemolytic activity in fresh human red blood cells [55]. 


\section{Conclusions}

This work describes, for the first time, the fabrication of non-crosslinked electrospun fibres of an ELR-based material functionalized with an antimicrobial peptide. It is noteworthy that the electrospun fibre mats were stable at the handling temperature, even in solution, which contrasts with previous works with electrospun fibres of ELRs. This avoids the use of crosslinking procedures that may react with fundamental amino acids of the antimicrobial domain thus potentially affecting its activity. We also provide, for the first time, a comparison of the thermal degradation profile and kinetics of materials obtained by two different processing techniques (electrospinning and solvent casting), using the same polymeric protein-based material. In this regard, the CM4-A200 fibre mats proved to be more thermally stable than the films, showing a higher thermal degradation activation energy.

The electrospun materials displayed a high antimicrobial activity against clinically relevant Gram-negative and Gram-positive bacteria, and proved to be non-cytotoxic in in vitro cultures of human keratinocytes and normal skin fibroblasts.

This work represents a major advance for the development and application of proteinbased materials suitable for biomedical applications, especially those requiring antimicrobial properties such as in the prevention of skin wound infections. For instance, as wound dressings for burn healing and skin reconstruction in which antibacterial properties are of utmost importance. Further, it also contributes to understand and expand the applicability of recombinant protein-based materials by providing valuable information on the thermal properties of the different processed CM4-A200 materials, an important parameter in materials science.

\section{Acknowledgments}

This work was financed by the strategic programme UID/BIA/04050/2013 (POCI-010145-FEDER-007569) funded by national funds through Fundação para a Ciência e a Tecnologia (FCT) and by the ERDF through the COMPETE2020 - Programa Operacional Competitividade e Internacionalização (POCI). The present work was also supported by FCT within the ERA-NET IB, project FunBioPlas with grant number ERA-IB-15-089 and FCT reference ERA-IB-2-6/0004/2014. AC and RM acknowledge FCT for SFRH/BD/75882/2011 and SFRH-BPD/86470/2012 grants, respectively. This 
article is a result of the project EcoAgriFood (NORTE-01-0145-FEDER-000009), supported by Norte Portugal Regional Operational Programme (NORTE 2020), under the PORTUGAL 2020 Partnership Agreement, through the European Regional Development Fund (ERDF). Authors also acknowledge the Spanish Minister of Economy and Competitiveness (MAT2013-41723-R, MAT2013- 42473-R and MAT2012-38043) and Junta de Castilla y León-JCyL (VA244U13, VA313U14), Spain.

\section{References}

[1] Casal M, Cunha AM, Machado R. Future Trends for Recombinant Protein-Based Polymers: The Case Study of Development and Application of Silk-Elastin-Like Polymers. Bio-Based Plastics: John Wiley \& Sons Ltd; 2013. p. 311-29.

[2] Margarida Pereira A, Machado R, da Costa A, Ribeiro A, Collins T, Gomes AC, et al. Silk-based biomaterials functionalized with fibronectin type II promotes cell adhesion. Acta Biomaterialia.

[3] Araujo R, Silva C, Machado R, Casal M, Cunha AM, Rodriguez-Cabello JC, et al. Proteolytic enzyme engineering: a tool for wool. Biomacromolecules 2009;10:1655-61. [4] da Costa A, Machado R, Ribeiro A, Collins T, Thiagarajan V, Neves-Petersen MT, et al. Development of Elastin-Like Recombinamer Films with Antimicrobial Activity. Biomacromolecules 2015;16:625-35.

[5] Punet X, Mauchauffé R, Giannotti MI, Rodríguez-Cabello JC, Sanz F, Engel E, et al. Enhanced Cell-Material Interactions through the Biofunctionalization of Polymeric Surfaces with Engineered Peptides. Biomacromolecules 2013;14:2690-702.

[6] Girotti A, Fernandez-Colino A, Lopez IM, Rodriguez-Cabello JC, Arias FJ. Elastinlike recombinamers: biosynthetic strategies and biotechnological applications. Biotechnol J 2011;6:1174-86.

[7] MacEwan SR, Chilkoti A. Elastin-like polypeptides: biomedical applications of tunable biopolymers. Biopolymers 2010;94:60-77.

[8] Nettles DL, Chilkoti A, Setton LA. Applications of elastin-like polypeptides in tissue engineering. Adv Drug Deliv Rev 2010.

[9] Simnick AJ, Lim DW, Chow D, Chilkoti A. Biomedical and biotechnological applications of elastin-like polypeptides. Polymer Reviews 2007;47:121-54. 
[10] Nettles DL, Kitaoka K, Hanson NA, Flahiff CM, Mata BA, Hsu EW, et al. In situ crosslinking elastin-like polypeptide gels for application to articular cartilage repair in a goat osteochondral defect model. Tissue Engineering Part A 2008;14:1133-40.

[11] Benitez PL, Sweet JA, Fink H, Chennazhi KP, Nair SV, Enejder A, et al.

Sequence-specific crosslinking of electrospun, elastin-like protein preserves bioactivity and native-like mechanics. Advanced healthcare materials 2013;2:114-8.

[12] Lim DW, Nettles DL, Setton LA, Chilkoti A. Rapid cross-linking of elastin-like polypeptides with (hydroxymethyl)phosphines in aqueous solution. Biomacromolecules 2007;8:1463-70.

[13] Putzu M, Causa F, Nele V, de Torre IG, Rodriguez-Cabello JC, Netti PA. Elastinlike-recombinamers multilayered nanofibrous scaffolds for cardiovascular applications. Biofabrication 2016;8:045009.

[14] Garcia-Arevalo C, Pierna M, Girotti A, Arias FJ, Rodriguez-Cabello JC. A comparative study of cell behavior on different energetic and bioactive polymeric surfaces made from elastin-like recombinamers. Soft Matter 2012;8:3239-49.

[15] Lee S, Kim J-S, Chu HS, Kim G-W, Won J-I, Jang J-H. Electrospun nanofibrous scaffolds for controlled release of adeno-associated viral vectors. Acta Biomaterialia 2011;7:3868-76.

[16] Nagapudi K, Brinkman WT, Leisen JE, Huang L, McMillan RA, Apkarian RP, et al. Photomediated solid-state cross-linking of an elastin-mimetic recombinant protein polymer. Macromolecules 2002;35:1730-7.

[17] Machado R, Ribeiro AJ, Padrao J, Silva D, Nobre A, Teixeira JA, et al. Exploiting the sequence of naturally occurring elastin: Construction, production and characterization of a recombinant thermoplastic protein-based polymer. J Nano Res 2009;6:133-45.

[18] Machado R, Bessa PC, Reis RL, Rodriguez-Cabello JC, Casal M. Elastin-based nanoparticles for delivery of bone morphogenetic proteins2012.

[19] Bessa PC, Machado R, Nurnberger S, Dopler D, Banerjee A, Cunha AM, et al. Thermoresponsive self-assembled elastin-based nanoparticles for delivery of BMPs. J Control Release 2010;142:312-8.

[20] Griffith M, Islam MM, Edin J, Papapavlou G, Buznyk O, Patra HK. The Quest for Anti-inflammatory and Anti-infective Biomaterials in Clinical Translation. Frontiers in bioengineering and biotechnology 2016;4:71. 
[21] Fernandes P, Martens E. Antibiotics in Late Clinical Development. Biochem Pharmacol 2016.

[22] Pendleton JN, Gorman SP, Gilmore BF. Clinical relevance of the ESKAPE pathogens. Expert Rev Anti Infect Ther 2013;11:297-308.

[23] Maragakis LL, Perencevich EN, Cosgrove SE. Clinical and economic burden of antimicrobial resistance. Expert Rev Anti-Infe 2008;6:751-63.

[24] Akova M. Epidemiology of antimicrobial resistance in bloodstream infections. Virulence 2016;7:252-66.

[25] May M. Drug development: Time for teamwork. Nature 2014;509:S4-5.

[26] Brogden KA. Antimicrobial peptides: pore formers or metabolic inhibitors in bacteria? Nat Rev Microbiol 2005;3:238-50.

[27] Lam SJ, O'Brien-Simpson NM, Pantarat N, Sulistio A, Wong EH, Chen YY, et al. Combating multidrug-resistant Gram-negative bacteria with structurally nanoengineered antimicrobial peptide polymers. Nature microbiology 2016;1:16162.

[28] Rios AC, Moutinho CG, Pinto FC, Del Fiol FS, Jozala A, Chaud MV, et al. Alternatives to overcoming bacterial resistances: State-of-the-art. Microbiol Res 2016;191:51-80.

[29] Matsuzaki K. Control of cell selectivity of antimicrobial peptides. Biochim Biophys Acta 2009;1788:1687-92.

[30] Lai Y, Gallo RL. AMPed up immunity: how antimicrobial peptides have multiple roles in immune defense. Trends Immunol 2009;30:131-41.

[31] Li BC, Zhang SQ, Dan WB, Chen YQ, Cao P. Expression in Escherichia coli and purification of bioactive antibacterial peptide ABP-CM4 from the Chinese silk worm, Bombyx mori. Biotechnol Lett 2007;29:1031-6.

[32] Rasband WS. ImageJ, U. S. National Institutes of Health, Bethesda, Maryland, USA, http://imagej.nih.gov/ij/. 1997-2011.

[33] Barth A. Infrared spectroscopy of proteins. Biochimica et Biophysica Acta (BBA) Bioenergetics 2007;1767:1073-101.

[34] Kong J, Yu S. Fourier transform infrared spectroscopic analysis of protein secondary structures. Acta Biochim Biophys Sin (Shanghai) 2007;39:549-59. [35] Machado R, da Costa A, Sencadas V, Pereira AM, Collins T, Rodriguez-Cabello JC, et al. Exploring the Properties of Genetically Engineered Silk-Elastin-Like Protein Films. Macromolecular Bioscience 2015;15:1698-709. 
[36] Dandurand J, Samouillan V, Lacoste-Ferre MH, Lacabanne C, Bochicchio B, Pepe

A. Conformational and thermal characterization of a synthetic peptidic fragment inspired from human tropoelastin: Signature of the amyloid fibers. Pathol Biol (Paris) 2014;62:100-7.

[37] Kissinger HE. Variation of Peak Temperature with Heating Rate in Differential Thermal Analysis. J Res Nat Bur Stand 1956;57:217-21.

[38] Kissinger HE. Reaction Kinetics in Differential Thermal Analysis. Analytical Chemistry 1957;29:1702-6.

[39] Sencadas V, Costa CM, Botelho G, Caparros C, Ribeiro C, Gomez-Ribelles JL, et al. Thermal Properties of Electrospun Poly(Lactic Acid) Membranes. J Macromol Sci B 2012;51:411-24.

[40] Bhardwaj N, Kundu SC. Electrospinning: a fascinating fiber fabrication technique. Biotechnol Adv 2010;28:325-47.

[41] Siemann U. Solvent cast technology - a versatile tool for thin film production. Progress in Colloid and Polymer Science 2005;130:14.

[42] Hu X, Lu Q, Sun L, Cebe P, Wang X, Zhang X, et al. Biomaterials from ultrasonication-induced silk fibroin-hyaluronic acid hydrogels. Biomacromolecules 2010;11:3178-88.

[43] Um IC, Kweon HY, Park YH, Hudson S. Structural characteristics and properties of the regenerated silk fibroin prepared from formic acid. Int J Biol Macromol 2001;29:91-7.

[44] Zhai Y, Cui FZ. Recombinant human-like collagen directed growth of hydroxyapatite nanocrystals. J Cryst Growth 2006;291:202-6.

[45] Kopinke FD, Mackenzie K. Mechanistic aspects of the thermal degradation of poly(lactic acid) and poly(beta-hydroxybutyric acid). J Anal Appl Pyrol 1997;40-1:4353.

[46] Buckton G, Beezer AE. The Relationship between Particle-Size and Solubility. Int J Pharm 1992;82:R7-R10.

[47] Machado R, da Costa A, Sencadas V, Garcia-Arévalo C, M. Costa C, Padrão J, et al. Electrospun silk-elastin-like fibre mats for tissue engineering applications.

Biomedical Materials 2013;8:065009.

[48] Serra R, Grande R, Butrico L, Rossi A, Settimio UF, Caroleo B, et al. Chronic wound infections: the role of Pseudomonas aeruginosa and Staphylococcus aureus. Expert Rev Anti-Infe 2015;13:605-13. 
[49] Esposito S, Noviello S, Leone S. Epidemiology and microbiology of skin and soft tissue infections. Current Opinion in Infectious Diseases 2016;29:109-15.

[50] Guillamet CV, Kollef MH. How to stratify patients at risk for resistant bugs in skin and soft tissue infections? Current Opinion in Infectious Diseases 2016;29:116-23. [51] Jenssen H, Hamill P, Hancock RE. Peptide antimicrobial agents. Clin Microbiol Rev 2006;19:491-511.

[52] Li JF, Zhang J, Xu XZ, Han YY, Cui XW, Chen YQ, et al. The antibacterial peptide ABP-CM4: the current state of its production and applications. Amino Acids 2012;42:2393-402.

[53] Rincon AC, Molina-Martinez IT, de Las Heras B, Alonso M, Bailez C, RodriguezCabello JC, et al. Biocompatibility of elastin-like polymer poly(VPAVG) microparticles: in vitro and in vivo studies. Journal of Biomedical Materials Research Part A 2006;78A:343-51.

[54] Massodi I, Raucher D. A thermally responsive Tat-elastin-like polypeptide fusion protein induces membrane leakage, apoptosis, and cell death in human breast cancer cells. Journal of Drug Targeting 2007;15:611-22.

[55] Chen YQ, Min C, Sang M, Han YY, Ma X, Xue XQ, et al. A cationic amphiphilic peptide ABP-CM4 exhibits selective cytotoxicity against leukemia cells. Peptides 2010;31:1504-10. 
a)

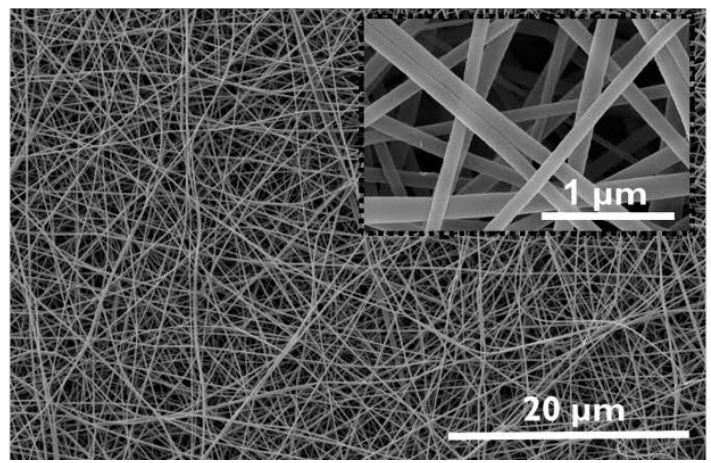

b)

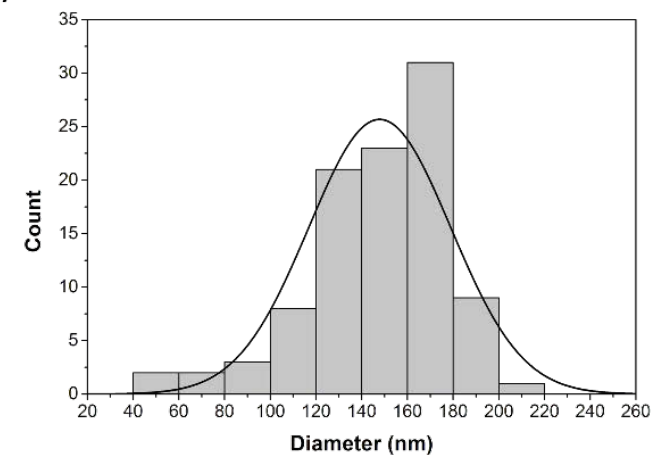

Figure 1 - SEM micrographs of CM4-A200 fibres (a) and corresponding histogram with diameter distribution (b). A normal distribution curve was applied in the histogram.

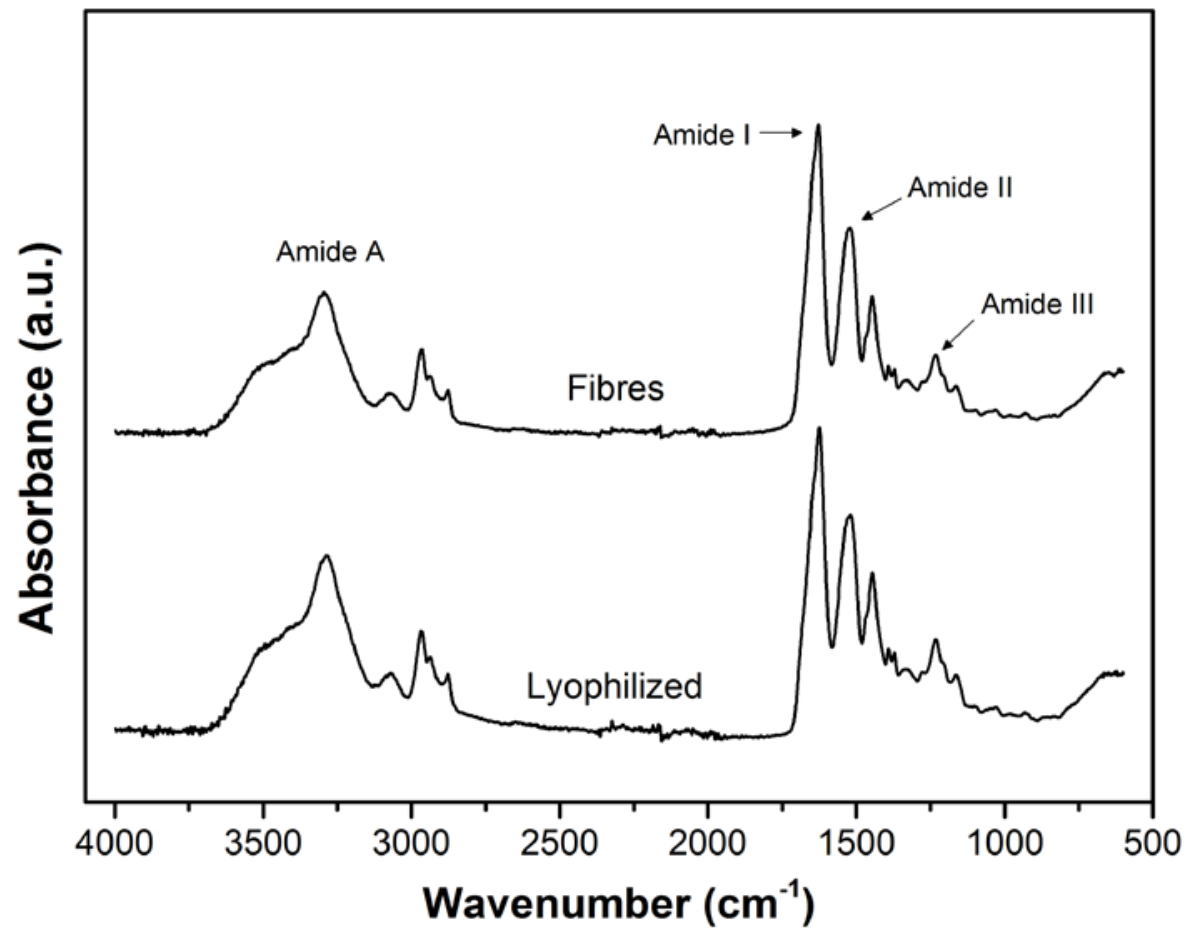

Figure 2: Infrared spectra of electrospun and lyophilized samples showing the characteristic amide absorption bands. 
a)

b)
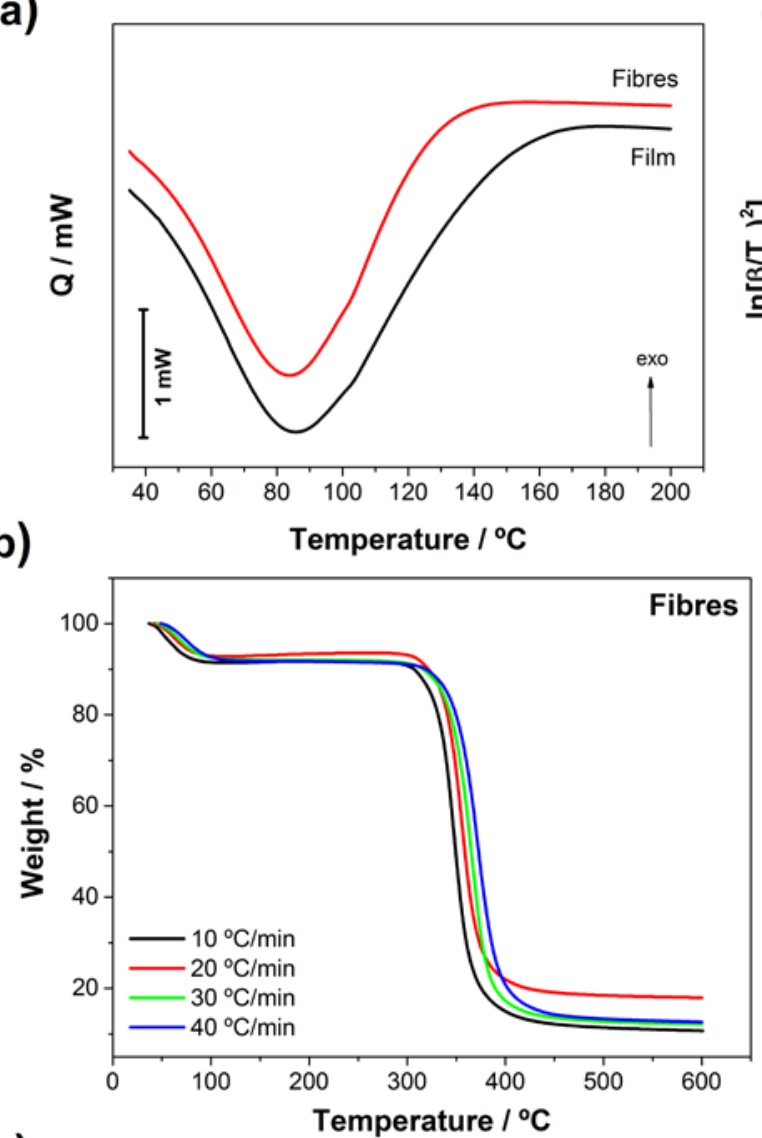

c)

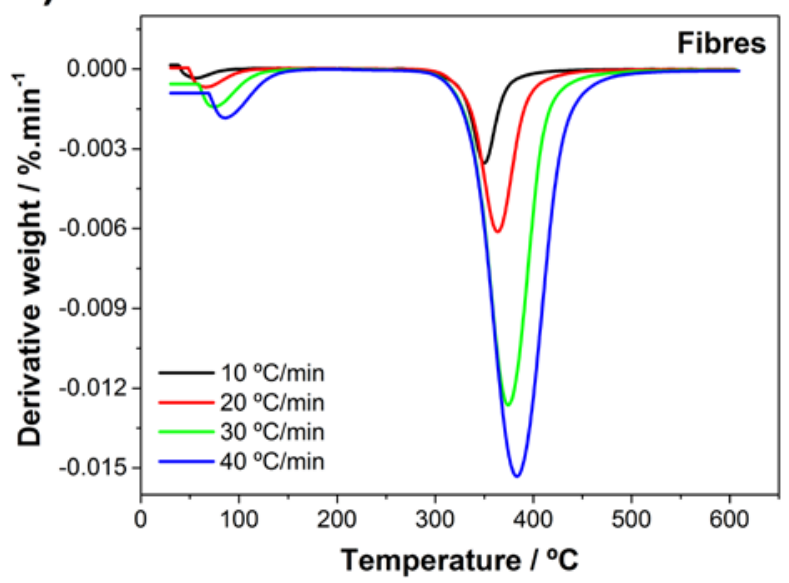

d)
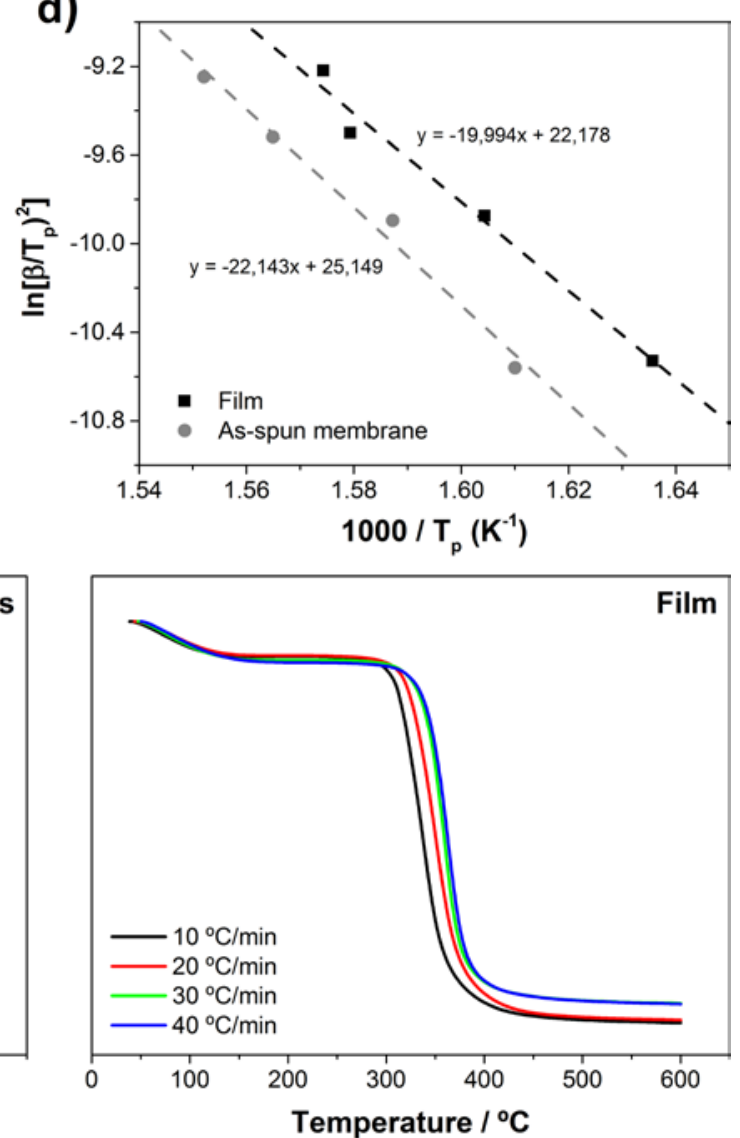

Temperature $/{ }^{\circ} \mathrm{C}$

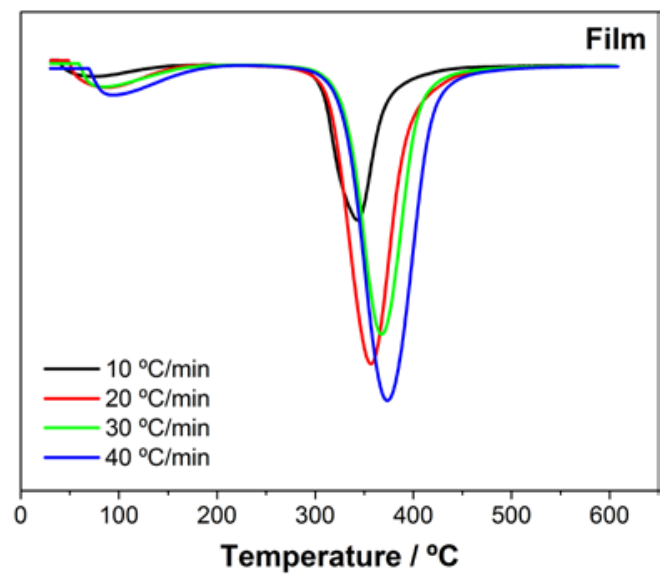

Figure 3: Thermograms obtained by DSC (a) and TGA (b) for the CM4-A200 materials produced by electrospinning and solvent casting. The derivative thermogravimetric curve (c) allows to distinguish the point at which the weight loss is more evident. Coloured lines represent the different heating rates utilized: black -10 ${ }^{\circ} \mathrm{C} \cdot \mathrm{min}^{-1}$; red $-20^{\circ} \mathrm{C} \cdot \mathrm{min}^{-1}$; green $-30^{\circ} \mathrm{C} \cdot \mathrm{min}^{-1}$; blue $-40^{\circ} \mathrm{C} \cdot \mathrm{min}^{-1}$. The activation energy for each material was determined by the slope of the Kissinger plot (d) multiplied by the gas constant. 
a)

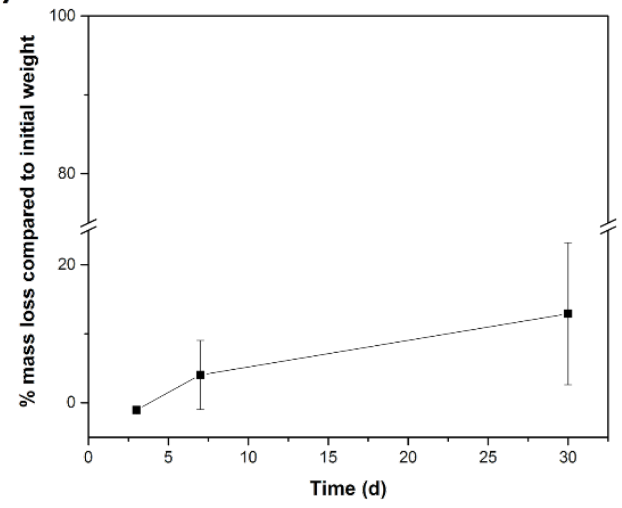

b)

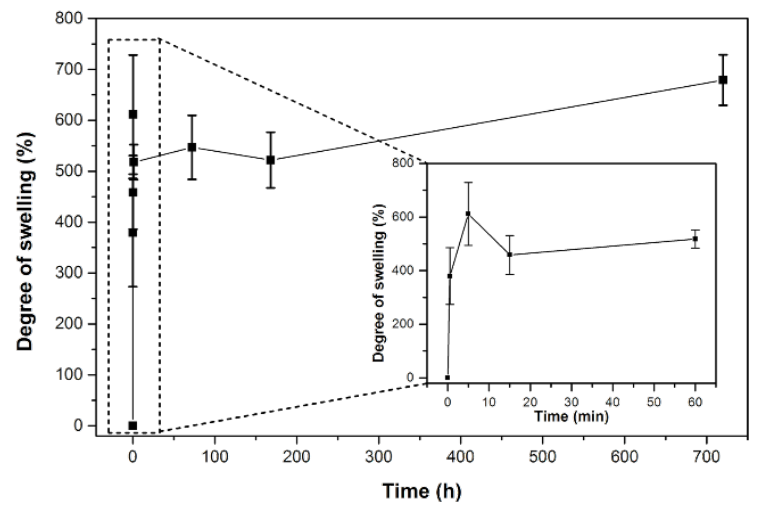

Figure 4. In vitro hydrolytic degradation profile (a) and degree of swelling (b) for CM4-A200 fibres immersed in PBS for 30 days at $37^{\circ} \mathrm{C}$. The inset in (b) represents the degree of swelling obtained during the first $60 \mathrm{~min}$ of immersion.

a)

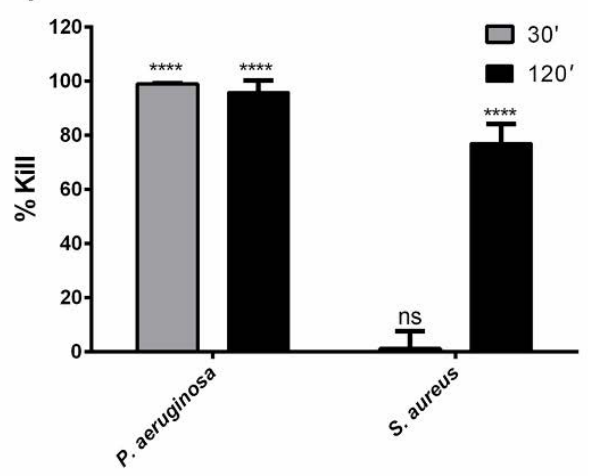

b)

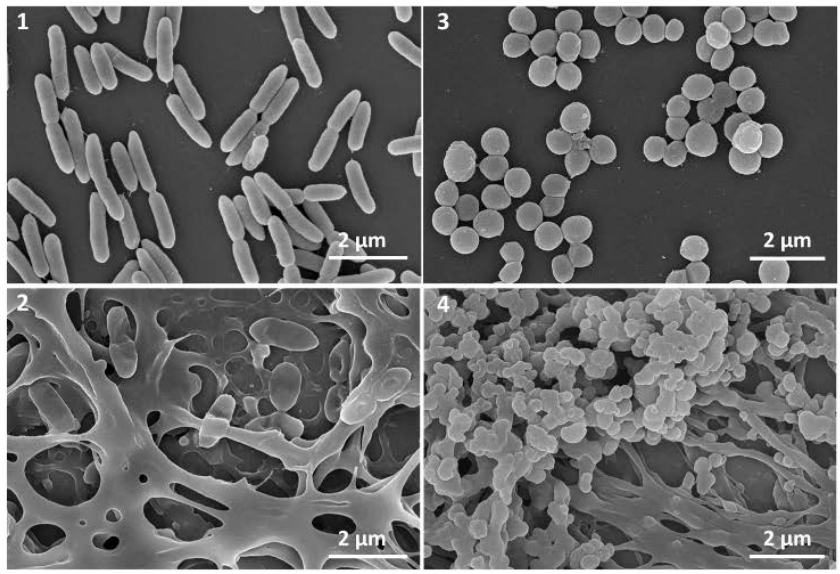

Figure 5 - 


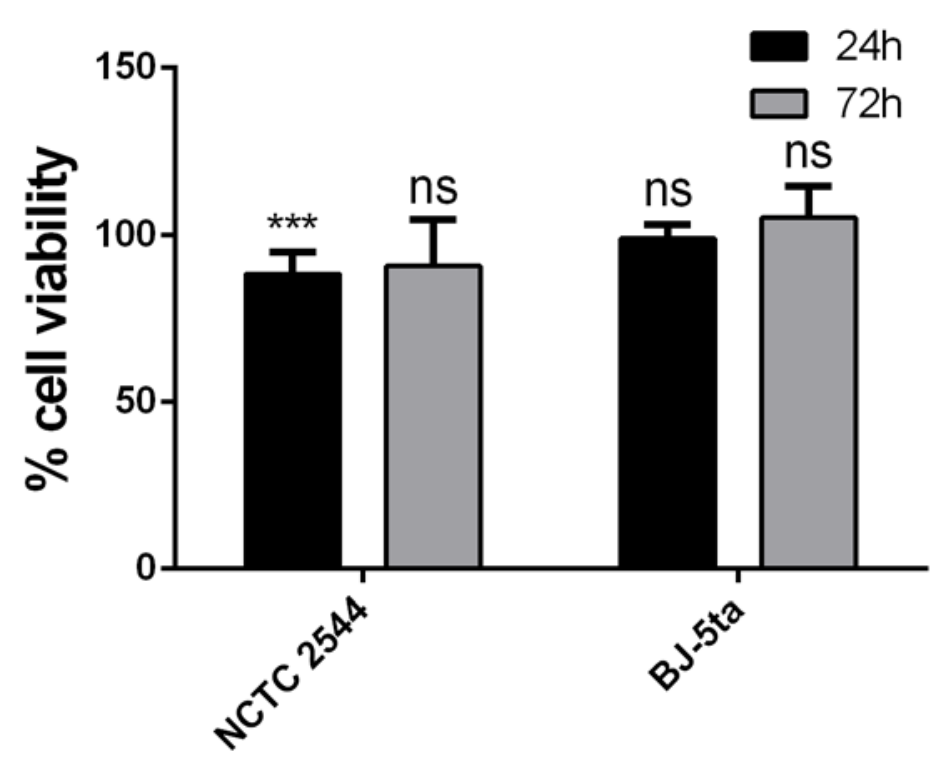

Figure 6 - Cytotoxicity evaluation of CM4-A200 fibre mats. Indirect contact viability assay was performed on normal human skin fibroblasts (BJ-5ta cell line) and human keratinocytes (NCTC 2544 cell line) using the MTS assay and represented as \% cell viability related to the untreated control; bars represent means \pm SD (ns, nonsignificant, $* * * \mathrm{p} \leq 0.001)$. 